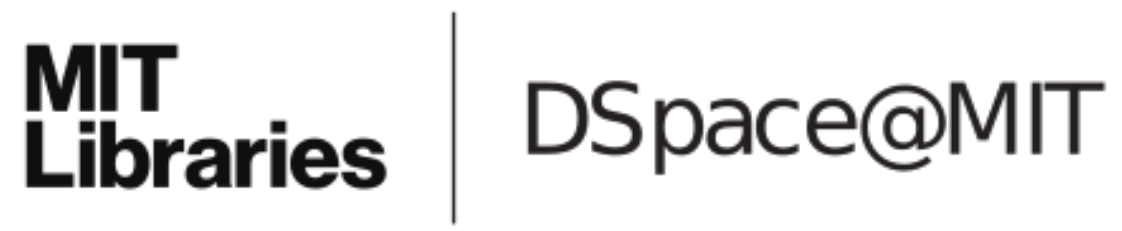

\author{
MIT Open Access Articles
}

Probing carbon isotope effects on the Raman spectra of
graphene with different [superscript 13]C concentrations

The MIT Faculty has made this article openly available. Please share how this access benefits you. Your story matters.

Citation: Carvalho, Bruno R., et al. "Probing carbon isotope effects on the Raman spectra of graphene with different [superscript 13]C concentrations." Phys. Rev. B 92, 125406 (September 2015). ( 2015 American Physical Society

As Published: http://dx.doi.org/10.1103/PhysRevB.92.125406

Publisher: American Physical Society

Persistent URL: http://hdl.handle.net/1721.1/98418

Version: Final published version: final published article, as it appeared in a journal, conference proceedings, or other formally published context

Terms of Use: Article is made available in accordance with the publisher's policy and may be subject to US copyright law. Please refer to the publisher's site for terms of use. 


\title{
Probing carbon isotope effects on the Raman spectra of graphene with different ${ }^{13} \mathrm{C}$ concentrations
}

\author{
Bruno R. Carvalho, ${ }^{1, *}$ Yufeng Hao, ${ }^{2}$ Ariete Righi, ${ }^{1}$ Joaquin F. Rodriguez-Nieva, ${ }^{3}$ Luigi Colombo, ${ }^{4}$ Rodney S. Ruoff, ${ }^{2,5,6}$ \\ Marcos A. Pimenta, ${ }^{1}$ and Cristiano Fantini ${ }^{1, \dagger}$ \\ ${ }^{1}$ Departamento de Física, Universidade Federal de Minas Gerais, 30123-970 Belo Horizonte, MG, Brazil \\ ${ }^{2}$ Department of Mechanical Engineering and the Materials Science and Engineering Program, The University of Texas at Austin, \\ 1 University Station C2200, Austin, Texas 78712-0292, USA \\ ${ }^{3}$ Department of Physics, Massachusetts Institute of Technology, Cambridge, Massachusetts 02139, USA \\ ${ }^{4}$ Texas Instruments Incorporated, 13121 TI Boulevard, MS-365 Dallas, Texas 75243, USA \\ ${ }^{5}$ Center for Multidimensional Carbon Materials, Institute for Basic Science (IBS), Ulsan 689-798, Republic of Korea \\ ${ }^{6}$ Department of Chemistry, Ulsan National Institute of Science and Technology (UNIST), Ulsan 689-798, Republic of Korea
}

(Received 16 June 2015; published 8 September 2015)

\begin{abstract}
A resonance Raman study of graphene samples with different ${ }^{13} \mathrm{C}$ isotopic concentrations and using different laser excitation energies is presented. The main Raman peaks $\left(D, G, G^{*}\right.$, and $\left.2 D\right)$ of graphene were measured and the dependence of their frequencies on the isotope atomic mass follows a simple harmonic oscillator relation. The $G^{*}$ and $2 D$ double-resonance peak positions were measured as a function of the laser energy, and we observed that the slopes of the laser energy dependence are the same independently of isotope concentration. This result shows that isotopic substitution does not alter the electron and phonon dispersions near the $K$ point of the graphene Brillouin zone. From the linewidth of $G$ and $2 D$ Raman peaks, we have also obtained a dependence of the phonon lifetime on the ${ }^{13} \mathrm{C}$ isotope concentration.
\end{abstract}

DOI: 10.1103/PhysRevB.92.125406

PACS number(s): 63.20.kd, 78.30.-j, 78.30.Na, 78.66.Tr

\section{INTRODUCTION}

Graphene is a two-dimensional material composed of a single layer of carbon atoms, and has been intensively studied due to its remarkable physical properties and large number of possible technological applications [1-3]. Significant efforts have been devoted to produce and study isotope-enriched graphene-related materials [4-13]. For instance, isotope labeling provided direct evidence that the growth mechanism of graphene is substrate dependent and, furthermore, shed light on the rational design of chemical vapor deposition (CVD) synthesis methods [4-8]. In addition, growing graphene with mixed carbon isotopes sources also enables tuning the properties of graphene in a highly selective manner. Despite the fact that isotope mixing does not affect the electronic or chemical properties of graphene $[14,15]$, their thermal and vibrational properties are sensitive to the mass of carbon atoms [16].

Raman spectroscopy has become a prominent nondestructive technique to study the vibrational properties of carbonrelated materials [17]. In particular, Raman spectroscopy is ideally used to probe isotope-doped graphene due to its sensitivity to the mass of constituent carbon atoms $[14,15]$. Resonance Raman spectroscopy provides additional information, such as electronic and phonon dispersion near the $K$ point of graphene [17].

Previous work on isotope-enriched samples of graphene and single-walled carbon nanotubes (SWCNTs), produced by different fabrication methods, has provided valuable information about their vibrational and electronic properties [14]. For instance, Miyauchi et al. [10] used pure ${ }^{12} \mathrm{C}$ and ${ }^{13} \mathrm{C}$ SWNTs and assigned phonon-assisted peaks in the photoluminescence spectra of nanotubes. In the case of SWCNTs, a redshift of the $G$ band when the ${ }^{13} \mathrm{C}$ concentration is increased

\footnotetext{
*brunorc@ fisica.ufmg.br

†fantini@fisica.ufmg.br
}

has been reported [10], and a dependence of the $G$ band linewidth on the isotope concentration was observed [14]. Studies of bilayer graphene, where one layer is composed of ${ }^{12} \mathrm{C}$ atoms and the other of ${ }^{13} \mathrm{C}$ atoms, were performed, thus allowing the investigation of interlayer interactions in the Raman scattering process $[11,15]$. It was also observed in twisted bilayer graphene that the $G$ band intensity is enhanced in regions of the sample where the separation in energy of the van Hove singularities in the density of states, which are induced by the twisted electronic band structure, match the laser excitation energy [15]. Other authors have suggested that the $G$ band resonance is closely related to the optically allowed electronic transition in twisted bilayer graphene $[18,19]$. Bernard et al. [13] identified several weak Raman peaks in isotope-enriched graphene, ascribed to the double-resonance Raman process, and obtained phonon dispersion for these mixed isotopic materials [13].

Additionally, several theoretical works were performed to explain the physical and transport properties of isotopeenriched samples, especially for graphene [16,20,21], singlewalled nanotubes [22], and boron nitride nanotubes [23]. In the aforementioned works, attention was paid primarily to the $G$ band. The behavior of other Raman features under isotope doping, however, remained unexplored.

In this paper we present a resonance Raman spectroscopy study, using six different laser excitation lines, in samples of isotope-enriched graphene synthesized by the CVD technique [5], with different ${ }^{13} \mathrm{C}$ concentrations of $0.01 \%, 25 \%$, $50 \%, 75 \%$, and $100 \%$. The focus of this work is to understand the effect of isotopic substitution on the position and linewidth of the main Raman features ( $D, G, G^{*}$, and $2 D$ bands). The analysis of the $2 D$ Raman peak as a function of the ${ }^{13} \mathrm{C}$ concentration and laser energy allowed us to conclude that the slopes of the electronic and phonon dispersions near the $K$ point are not affected by the isotope enrichment of the graphene samples. Finally, we present results of the phonon mean free path as a function of the isotopic ratio. 


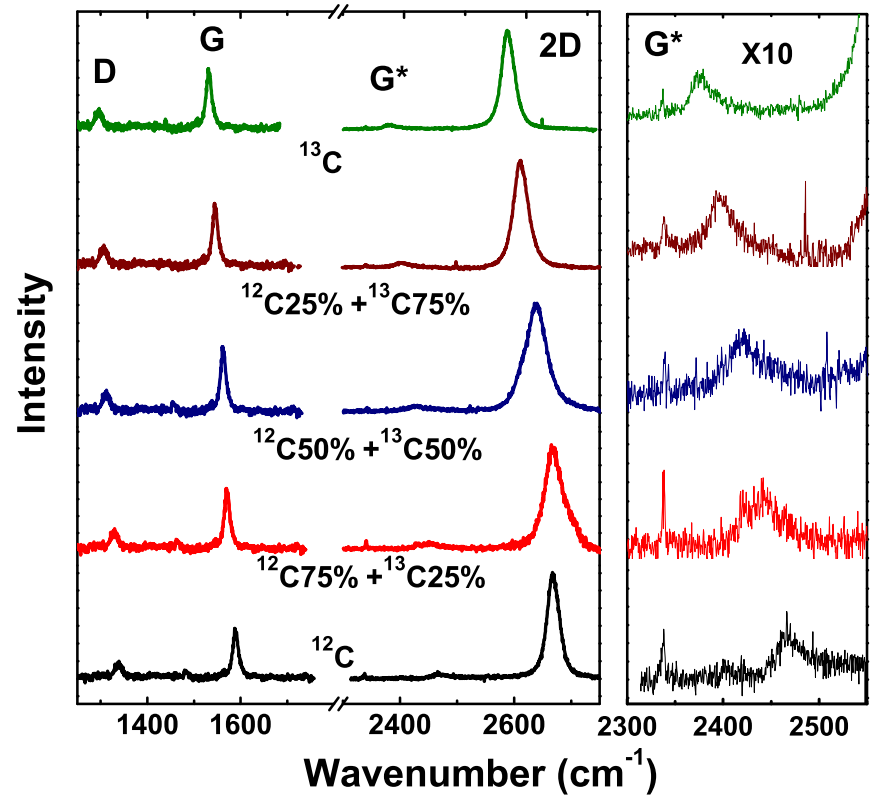

FIG. 1. (Color online) Raman spectra of the main graphene features ( $D, G, G^{*}$, and $2 D$ bands) for different amounts of the ${ }^{13} \mathrm{C}$ isotope. The right panels correspond to $10 \times$ zooming of the $G^{*}$ band. The spectra were recorded using $2.18 \mathrm{eV}$ laser excitation energy.

\section{MATERIALS AND METHODS}

Samples of single-layer graphene with different isotope concentrations were produced by the CVD method, on a $\mathrm{Cu}$ substrate at high temperature using the procedure reported in Ref. [5]. Raman spectra were measured at room temperature with a triple monochromator (DILOR XY) and Horiba T64000 spectrometers equipped with a liquid- $\mathrm{N}_{2}$-cooled chargecoupled device (CCD). The spot size was $\sim 1 \mu \mathrm{m}$ for a $100 \times$ objective, and the power was kept at $\sim 1.0 \mathrm{~mW}$ to avoid heating and damage of the sample. The excitations energies were $1.92 \mathrm{eV}(647.1 \mathrm{~nm}), 2.18 \mathrm{eV}(568.2 \mathrm{~nm}), 2.41 \mathrm{eV}(514.5 \mathrm{~nm})$, $2.54 \mathrm{eV}(488 \mathrm{~nm})$, and $2.71 \mathrm{eV}(457.9 \mathrm{~nm})$ from an $\mathrm{Ar}-\mathrm{Kr}$ laser and $2.81 \mathrm{eV}(441 \mathrm{~nm})$ from the $\mathrm{He}-\mathrm{Cd}$ laser.

\section{RESULTS AND DISCUSSION}

Figure 1 shows the Raman spectra of a single-layer graphene with five different ${ }^{13} \mathrm{C}$ concentrations $(0.01 \%, 25 \%$, $50 \%, 75 \%$, and $100 \%$ ), obtained with a $2.18 \mathrm{eV}$ laser excitation energy and displaying the main Raman features (the $D, G, G^{*}$, and $2 D$ bands). The $D$ mode appears around $1350 \mathrm{~cm}^{-1}$ for the pure ${ }^{12} \mathrm{C}$ sample and consists in a breathing mode of six-atom rings, activated via a defect in the lattice. It originates from one in-plane transverse optical (iTO) phonon around the $K$ or $K^{\prime}$ points in the first Brillouin zone and involves an intervalley double-resonance (DR) process. The $G$ band appears around $1580 \mathrm{~cm}^{-1}$ for the pure ${ }^{12} \mathrm{C}$ sample and corresponds to the $E_{2 g}$ phonon mode at the Brillouin zone center. The $G^{*}$ and $2 D$ bands for pure ${ }^{12} \mathrm{C}$ are around 2450 and $2675 \mathrm{~cm}^{-1}$, respectively. Both are second-order modes and originate from intervalley DR processes. The $G^{*}$ band involves one iTO and one longitudinal acoustic (LA) phonon whereas the $2 D$ band involves two iTO phonons near the $K$ point.

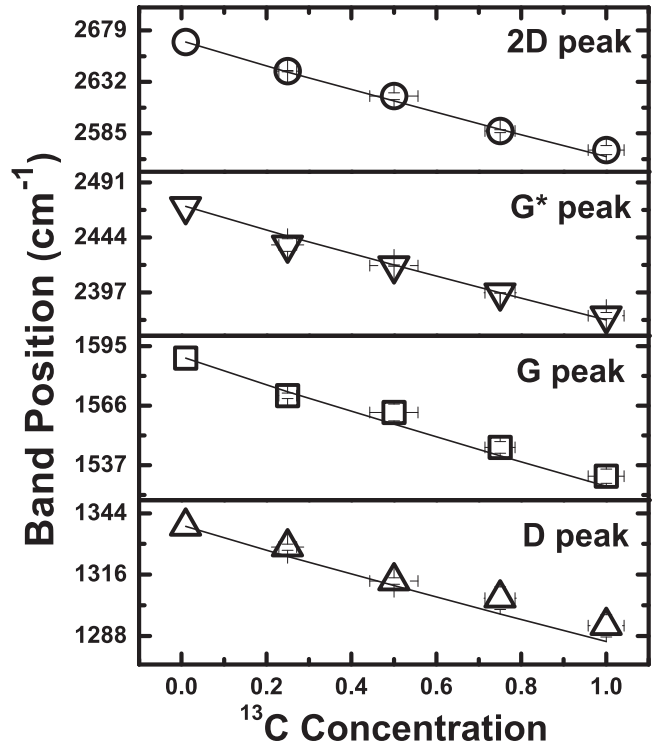

FIG. 2. From the bottom to top: $D, G, G^{*}$, and $2 D$ Raman peak positions as a function of the real ${ }^{13} \mathrm{C}$ isotopic-enriched content in the sample to the $2.18 \mathrm{eV}$ laser excitation energy. The symbols represent experimental points and the solid lines the estimated dependence obtained through Eq. (1).

As expected for isotope-enriched carbon materials $[4,5,11,14]$, the position of the Raman peaks decreases with increasing ${ }^{13} \mathrm{C}$ concentration, which is due to the mass difference between the ${ }^{12} \mathrm{C}$ and ${ }^{13} \mathrm{C}$ isotopes. Figure 2 depicts the Raman frequency of the bands shown in Fig. 1, as a function of the ${ }^{13} \mathrm{C}$ concentration. The straight lines correspond to the fit of the experimental data based on the simple harmonic oscillator model, considering that the frequency of the Raman bands is inversely proportional to the square root of the mean atomic mass, and is given by

$$
\omega(x)=\omega_{12} \mathrm{C} \sqrt{\frac{m_{0}}{m_{0}+x \Delta m}} .
$$

Here, $x$ is the number density of ${ }^{13} \mathrm{C}(0 \leqslant x \leqslant 1)$ in the enriched graphene samples, $\omega_{12_{\mathrm{C}}}$ represents the Raman frequency in the ${ }^{12} \mathrm{C}$ graphene sample, $m_{0}$ is the mass of ${ }^{12} \mathrm{C}$, and $\Delta m$ is the mass difference between the ${ }^{12} \mathrm{C}$ and ${ }^{13} \mathrm{C}$ isotope. Note that the dependence of the Raman frequencies on the concentration $x$ is very well explained by the model given by Eq. (1), showing that isotopic mixing does not affect the force constant, but only the mean atomic mass. The observed redshifts of the Raman features with increasing ${ }^{13} \mathrm{C}$ concentration are in good agreement with previous results reported in the literature [11,14].

The laser energy dependences of the $G^{*}$ and $2 D$ peaks are related to the Fermi velocity $v_{F}$ according to the following expressions [17],

$$
\begin{aligned}
& \frac{d \omega_{2 D}}{d E_{L}}=\frac{2}{\hbar v_{F}} \frac{d \omega_{\mathrm{iTO}}}{d q}, \\
& \frac{d \omega_{G^{*}}}{d E_{L}}=\frac{1}{\hbar v_{F}}\left(\frac{d \omega_{\mathrm{LA}}}{d q}+\frac{d \omega_{\mathrm{iTO}}}{d q}\right),
\end{aligned}
$$



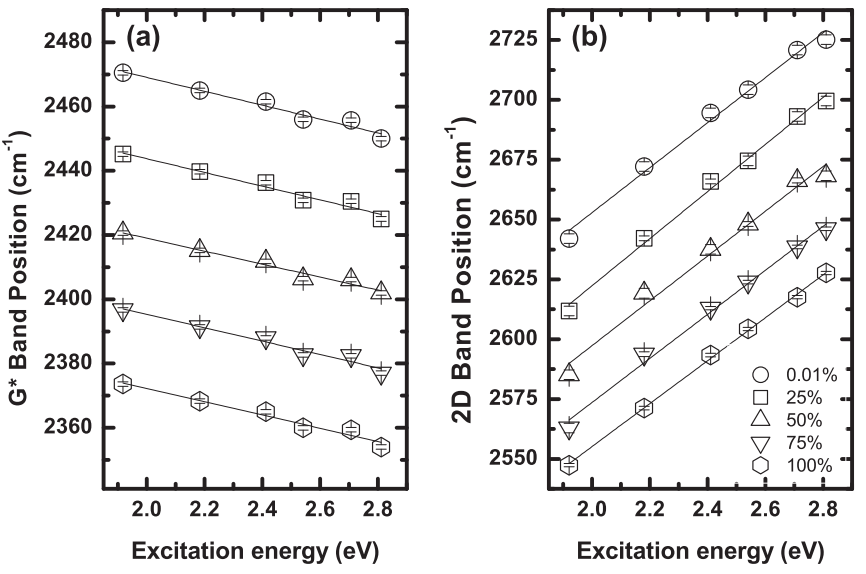

FIG. 3. The $G^{*}$ and $2 D$ Raman peak positions as a function of the different laser excitation energies. The solid lines represent their linear fitting functions.

where $\left(d \omega_{\mathrm{iTO}} / d q\right)$ and $\left(d \omega_{\mathrm{LA}} / d q\right)$ are the slopes of the iTO and LA phonon dispersions near the $K$ point. The slope of the dispersion of the double-resonance Raman bands with respect to the laser energy is therefore related to the phonon and Fermi velocities, respectively.

In order to study the effect of the isotopic substitution on the phonon and Fermi velocities, we analyze the laser frequency dependence of the $G^{*}$ and $2 D$ bands, shown in Fig. 3, for the five different ${ }^{13} \mathrm{C}$ isotopic concentrations $(0.01 \%, 25 \%, 50 \%$, $75 \%$, and $100 \%$ ). The dispersions were fitted by straight lines and the vertical shifts of these lines are only a consequence of the different mean atomic masses of these samples.

The slopes of the laser energy dependence of the $G^{*}$ and $2 D$ bands, shown in Table I, are roughly the same for all samples. As shown in Eqs. (2), these slopes are related to both the Fermi velocity and iTO and LA phonon dispersions near the $K$ point. Assuming that $v_{F}$ does not depend significantly on the isotope concentration, the iTO and LA phonon dispersions, near the $K$ point, are also invariant with isotope concentration.

By taking $v_{F}=1 \times 10^{6} \mathrm{~m} / \mathrm{s}$, we can obtain from Eq. (2) and Fig. 3 the phonon velocity of both iTO and LA phonon branches in the vicinity of the $K$ point for all samples investigated, and the results are presented in Table I. A similar behavior of the $2 D$ band dispersion was observed in a resonance Raman study of SWCNTs with different isotopic concentrations [14]. However, the $2 D$ band in SWCNTs depends on the chirality distribution of the $(n, m)$ nanotubes present in the sample, and therefore the results presented for
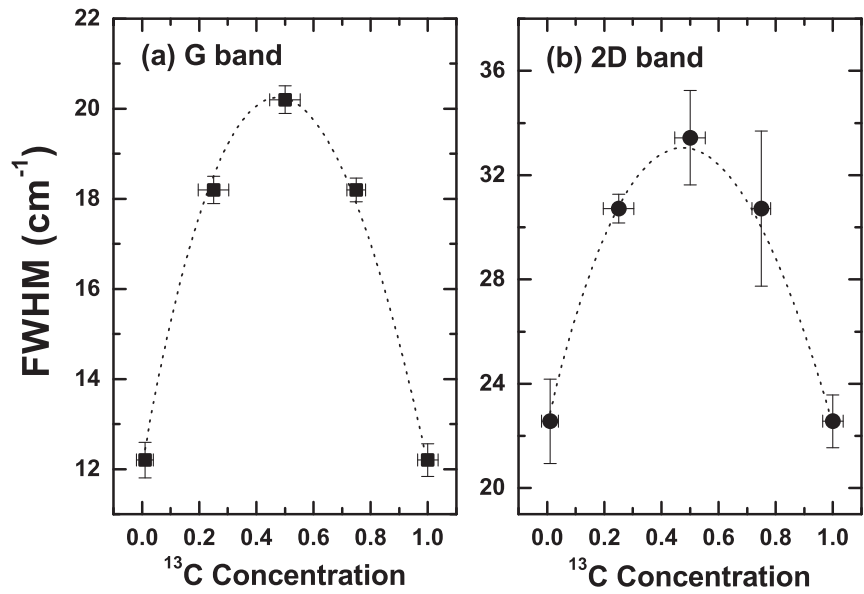

FIG. 4. (a) $G$ and (b) $2 D$ band linewidths as a function of the ${ }^{13} \mathrm{C}$ isotope-enriched concentration in the sample recorded at $2.18 \mathrm{eV}$ laser excitation energy. The curve was obtained through Eq. (2).

single-layer graphene in Fig. 3 are more fundamental. The results shown in Table I correspond to the phonon velocities with wave vectors that are probed by resonance Raman scattering in the visible range. The Kohn anomaly [24-26] might decrease the phonon velocity in the IR range. Anyway, our results, obtained with visible laser lines, demonstrate that the slope of the phonon dispersion in the visible range does not depend on the isotopic concentration.

Let us now discuss the dependence of the linewidths of the Raman bands as a function of the ${ }^{13} \mathrm{C}$ concentration. This dependence was already reported and explained for the case of the $G$ band in SWNTs [14]. However, the linewidth of the 2D band in SWNTs depends on the chirality distribution. We report here the behavior of the 2D linewidth with isotopic substitution in single-layer graphene.

Figures 4(a) and 4(b) depict the dependence of the full width at half maximum (FWHM) of the $G$ and $2 D$ bands on the ${ }^{13} \mathrm{C}$ concentration, respectively. Note that the FWHM of the $G$ and $2 D$ bands is the same for the two pure graphene samples with pure ${ }^{12} \mathrm{C}$ or ${ }^{13} \mathrm{C}$. However, the FWHM increases in the mixed samples, reaching its maximum value for $x=0.5$. The result for the $G$ band is similar to that reported for SWNTs [14], and we notice that the $2 D$ band FWHM exhibits the same behavior [Fig. 4(b)]. The experimental data shown in Fig. 4 were fitted using the expression below [20],

$$
\gamma_{G, 2 D}=\gamma_{e-\mathrm{ph}}^{\mathrm{expt}}+\gamma_{\mathrm{ph}-\mathrm{im}}^{\mathrm{expt}} \frac{f(x)}{f(0.5)},
$$

TABLE I. Slope of the $G^{*}$ and $2 D$ bands and phonon velocities for each amount of ${ }^{13} \mathrm{C}$ isotopic concentration. The error bars for the slope and phonon velocities represent the uncertainty associated with the standard error from the fitting process.

\begin{tabular}{|c|c|c|c|c|}
\hline & \multicolumn{2}{|c|}{ Slope $\left(\mathrm{cm}^{-1} / \mathrm{eV}\right)$} & \multicolumn{2}{|c|}{ Phonon velocities $\left(\times 10^{-3} v_{F}\right)$} \\
\hline & $d \omega_{2 D} / d E_{L}$ & $d \omega_{G^{*}} / d E_{L}$ & $d \omega_{\mathrm{iто}} / d q$ & $d \omega_{\mathrm{LA}} / d q$ \\
\hline Pure ${ }^{12} \mathrm{C}$ & $94 \pm 2$ & $-22 \pm 2$ & $5.6 \pm 0.1$ & $8.2 \pm 0.4$ \\
\hline${ }^{12} \mathrm{C}_{75 \%}+{ }^{13} \mathrm{C}_{25 \%}$ & $98 \pm 4$ & $-21 \pm 2$ & $5.9 \pm 0.2$ & $8.4 \pm 0.5$ \\
\hline${ }^{12} \mathrm{C}_{50 \%}+{ }^{13} \mathrm{C}_{50 \%}$ & $94 \pm 4$ & $-20 \pm 2$ & $5.6 \pm 0.2$ & $8.1 \pm 0.4$ \\
\hline${ }^{12} \mathrm{C}_{25 \%}+{ }^{13} \mathrm{C}_{75 \%}$ & $92 \pm 4$ & $-21 \pm 2$ & $5.5 \pm 0.2$ & $8.0 \pm 0.4$ \\
\hline Pure ${ }^{13} \mathrm{C}$ & $90 \pm 1$ & $-21 \pm 2$ & $5.4 \pm 0.1$ & $7.9 \pm 0.3$ \\
\hline
\end{tabular}


where $f(x)$ is a factor which contains all information about the density of impurities and is given by

$$
f(x)=\frac{x(1-x) \Delta m^{2}}{\left(m_{0}+x \Delta m\right)^{2}} \sqrt{\frac{m_{0}}{m_{0}+x \Delta m}} .
$$

Equation (3) estimates the contribution of the isotope impurities to the spectral width of the $G$ and $2 D$ Raman peaks, associated with the lifetime of the optical phonon mode. The value $\gamma_{\mathrm{ph} \text {-im }}^{\text {expt }}$ corresponds to the linewidth of the ${ }^{13} \mathrm{C}$ concentration for $x=0.5$.

The curves shown in Fig. 4 correspond to the best fit of the experimental data by Eq. (3), and the fitting parameters to the $G$ band are $\gamma_{e \text {-ph }}^{\text {expt }}=12.1 \pm 0.4 \mathrm{~cm}^{-1}$ and $\gamma_{\mathrm{ph} \text {-im }}^{\text {ext }}=8.2 \pm$ $0.4 \mathrm{~cm}^{-1}$. The values for the $2 D$ band are $\gamma_{e \text {-ph }}^{\text {expt }}=22.4 \pm$ $0.3 \mathrm{~cm}^{-1}$ and $\gamma_{\mathrm{ph}-\mathrm{im}}^{\mathrm{expt}}=10.6 \pm 0.3 \mathrm{~cm}^{-1}$. Since the spectral width $\gamma$ is related to the phonon lifetime $\gamma_{\text {ph-im }} \approx \hbar / \tau_{\text {ph-im }}$, we obtain the phonon lifetimes due to the ${ }^{13} \mathrm{C}$ concentration for both $G$ and $2 D$ bands for an isotopic concentration of $x=0.5$, which are 0.61 and $0.47 \mathrm{ps}$, respectively.

The observed dependence of the $G$ and $2 D$ FWHM on the concentration $x$ is a consequence of the decrease of the phonon lifetime in isotopic-doped graphene. When the isotopes are mixed, the translational symmetry is broken, causing a modification in the dynamics of the crystal lattice. The phonons are scattered into other phonon states, which ensures that some wave functions will be tightly localized in the direct space, resembling Anderson's localization wave function for a disordered crystal lattice [20]. The mean free path is related to the length of the localized states due to the phonon scattering by the isotope mixture. Therefore, the phonon mean free path decreases in the mixed samples and is minimum for isotope-enriched graphene with $x=0.5$.

\section{CONCLUSIONS}

In summary, in this paper we reported a resonance Raman study of isotope-enriched graphene containing different ${ }^{13} \mathrm{C}$ isotope concentrations. The dependence of the frequencies of the main Raman features of single-layer graphene on the ${ }^{13} \mathrm{C}$ concentration was reported and explained by a simple harmonic oscillator model, where the frequency is proportional to the inverse of the square root of the mean atomic mass. The dispersive behaviors of the $G^{*}$ and $2 D$ bands when changing the laser energy were measured for all pure and mixed samples, and no dependence of this dispersion on the ${ }^{13} \mathrm{C}$ isotope concentration was observed. This result suggests that the dispersion of iTO and LA phonons near the $K$ point is not significantly affected by isotopic substitution. Thus, the isotopic substitution is responsible for a shift in the phonon frequencies but does not affect the phonon dispersion slopes. Finally, it was observed that the isotopic mixture affects the bandwidth of the $G$ and $2 D$ bands, and the results can be explained by a theoretical model that considers the decrease of the mean free path and the lifetime of the phonons, due to scattering of phonons by different carbon isotopes in mixed samples.

\section{ACKNOWLEDGMENTS}

This work was supported by the Instituto Nacional de Ciência e Tecnologia (INCT) em Nanomateriais de Carbono (Brazil), Coordenação de Aperfeiçoamento de Pessoal de Nível Superior (CAPES, Brazil), Conselho Nacional de Desenvolvimento Cientifico e Tecnológico (CNPq, Brazil) and Fundação de Amparo à Pesquisa do Estado de Minas Gerais (FAPEMIG, Brazil), and the Southwest Academy of Nanotechnology (SWAN)/Nanoelectronics Research Initiative (NRI).
[1] K. S. Novoselov, A. K. Geim, S. V. Morozov, D. Jiang, Y. Zhang, S. V. Dubonos, I. V. Grigorieva, and A. A. Firsov, Science 306, 666 (2004).

[2] A. Geim, Nat. Mater. 6, 183 (2007).

[3] A. H. Castro Neto, F. Guinea, N. M. R. Peres, K. S. Novoselov, and A. K. Geim, Rev. Mod. Phys. 81, 109 (2009).

[4] X. S. Li, W. W. Cai, L. Colombo, and R. S. Ruoff, Nano Lett. 9, 4268 (2009).

[5] Y. Hao, M. S. Bharathi, L. Wang, Y. Liu, H. Chen, S. Nie, X. Wang, H. Chou, C. Tan, B. Fallahazad, H. Ramanarayan, C. W. Magnuson, E. Tutuc, B. I. Yakobson, K. F. McCarty, Y.-W. Zhang, P. Kim, J. Hone, L. Colombo, and R. S. Ruoff, Science 342, 720 (2013).

[6] S. Fan, L. Liu, and M. Liu, Nanotechnology 14, 1118 (2003).

[7] Q. Li, H. Chou, J.-H. Zhong, J.-Y. Liu, A. Dolocan, J. Zhang, Y. Zhou, R. S. Ruoff, S. Chen, and W. Cai, Nano Lett. 13, 486 (2013).

[8] W. Fang, A. L. Hsu, R. Caudillo, Y. Song, A. G. Birdwell, E. Zakar, M. Kalbac, M. Dubey, T. Palacios, M. S. Dresselhaus, P. T. Araujo, and J. Kong, Nano Lett. 13, 1541 (2013).

[9] F. Simon, Ch. Kramberger, R. Pfeiffer, H. Kuzmany, V. Zólyomi, J. Kürti, P. M. Singer, and H. Alloul, Phys. Rev. Lett. 95, 017401 (2005).
[10] Y. Miyauchi and S. Maruyama, Phys. Rev. B 74, 035415 (2006).

[11] M. Kalbac, H. Farhat, J. Kong, P. Janda, L. Kavan, and M. S. Dresselhaus, Nano Lett. 11, 1957 (2011).

[12] H. Watanabe, T. Koretsune, S. Nakashima, S. Saito, and S. Shikata, Phys. Rev. B 88, 205420 (2013).

[13] S. Bernard, E. Whiteway, V. Yu, D. G. Austing, and M. Hilke, Phys. Rev. B 86, 085409 (2012).

[14] S. D. Costa, C. Fantini, A. Righi, A. Bachmatiuk, M. H. Rümmeli, R. Saito, and M. A. Pimenta, Carbon 49, 4719 (2011).

[15] E. del Corro, M. Kalbac, C. Fantini, O. Frank, and M. A. Pimenta, Phys. Rev. B 88, 155436 (2013).

[16] S. Chen, Q. Wu, C. Mishra, J. Kang, H. Zhang, K. Cho, W. Cai, A. A. Balandin, and R. S. Ruoff, Nat. Mater. 11, 203 (2012).

[17] L. M. Malard, M. A. Pimenta, G. Dresselhaus, and M. S. Dresselhaus, Phys. Rep. 473, 51 (2009).

[18] J.-B. Wu, X. Zhang, M. Ijäs, W.-P. Han, X.-F. Qiao, X.-L. Li, D.-S. Jiang, A. C. Ferrari, and P.-H. Tan, Nat. Commun. 5, 5309 (2014).

[19] J.-B. Wu, Z.-X. Hu, X. Zhang, W.-P. Han, Y. Lu, W. Shi, X.-F. Qiao, M. Ijiäs, S. Milana, W. Ji, A. C. Ferrari, and P.-H. Tan, ACS Nano 9, 7440 (2015). 
[20] J. F. Rodriguez-Nieva, R. Saito, S. D. Costa, and M. S. Dresselhaus, Phys. Rev. B 85, 245406 (2012).

[21] H. Zhang, G. Lee, A. F. Fonseca, T. L. Borders, and K. Cho, J. Nanomater. 2010, 537657 (2010).

[22] T. Yamamoto, K. Sasaoka, and S. Watanabe, Phys. Rev. Lett. 106, 215503 (2011).

[23] I. Savić, N. Mingo, and D. A. Stewart, Phys. Rev. Lett. 101, 165502 (2008).
[24] D. L. Mafra, L. M. Malard, S. K. Doorn, H. Htoon, J. Nilsson, A. H. Castro Neto, and M. A. Pimenta, Phys. Rev. B 80, 241414(R) (2009).

[25] S. Piscanec, M. Lazzeri, F. Mauri, A. C. Ferrari, and J. Robertson, Phys. Rev. Lett. 93, 185503 (2004).

[26] M. Lazzeri, C. Attaccalite, L. Wirtz, and F. Mauri, Phys. Rev. B 78, 081406(R) (2008). 